\title{
FOUR DIFFERENT PRESENTATIONS OF WILSON'S DISEASE IN ONE FAMILY
}

\author{
Asbah Rahman, Qudratullah Malik*, Farooq Ikram* \\ Pak Emirates Military Hospital/National University of Medical Sciences (NUMS) Rawalpindi Pakistan, *Combined Military Hospital/National University of \\ Medical Sciences (NUMS) Rawalpindi Pakistan
}

\begin{abstract}
Wilson's disease (WD) is an important differential to consider in any child presenting with hepatic, neurological or ophthalmological manifestations of the disease. We report here 4 individuals of the same family: 2 paediatric and 2 adult patients with a spectrum of manifestations of the disease presenting to Pak Emirates Military Hospital and Combined Military Hospital Rawalpindi, Rawalpindi, from January 2019 and September 2020. The index case had neuro-wilson; the brother was diagnosed preemptively during screening; the father being completely asymptomatic despite markedly raised 24 hours urinary copper levels; and the paternal uncle being diagnosed after many years of manifesting hepatic symptoms. The purpose of this publication is to sensitize the readers to the usage of scoring tools such as the Leipzig score, the importance of regular follow-up and family screening of hereditary diseases. We would also like to highlight the possibility of missed diagnosis with serum Copper levels (S.Copper) which were within normal limits (WNL) in all 4 of our patients; and Serum ceruloplasmin (S.ceruloplasmin) levels which were within normal limits in 3 out of 4 of these patients, that are often used as screening tools for $\mathrm{WD}$.
\end{abstract}

Keywords: ATP7B, KF rings, Leipzig score, Unified wilson's disease rating scale, Wilson's disease.

This is an Open Access article distributed under the terms of the Creative Commons Attribution License (https://creativecommons.org/licenses/by-nc/4.0/), which permits unrestricted use, distribution, and reproduction in any medium, provided the original work is properly cited.

\section{INTRODUCTION}

WD was first described by British neurologist Samuel Wilson in 19121. It leads to excessive copper deposition in liver, brain, kidneys, cornea and cardiac muscles. Diagnosis is often missed because of its wide spectrum of presentation. Being autosomal recessive (AR) in origin, it may affect multiple individuals of the same family and family screening for homozygous or heterozygous carriers is essential. The worldwide incidence is 15-25 per million births, making mass screening an unpractical and uneconomical option ${ }^{2}$. Early diagnosis and timely initiation of treatment is mandatory for improving prognosis of patient. Treatment is multi-pronged consisting of copper chelating drugs, dietary modification, and competitive inhibition of copper absorption from the gut and replacement of fat soluble vitamins. Left untreated it may lead to cirrhosis of liver and irreversible worsening of neuropsychiatric symptoms as well as progressive involvement of other organ systems.

\section{Index case}

An 11-year-old boy reported to Pak Emirates Military Hospital, Rawalpindi in February 2019 with complaints of unintelligible speech and difficulty walking for the past 1 month. There was also history of difficulty in chewing food. On examination, the child had difficulty ambulating with ataxic gait and dysarth-

Correspondence: Dr Asbah Rahman, Resident Pediatrics, Pak Emirates Military Hospital, Rawalpindi Pakistan

Received: 16 Feb 2021; revised received: 26 Feb 2021; accepted: 07 May 2021 ria; however, no choreoform movements, intentional or resting tremors, past pointing or nystagmus was present. There was progressive stiffening of the first metacarpophalangeal joint of the right hand which had lead to dystonic flexion of the right thumb. He had an enlarged liver that was palpable $4 \mathrm{~cm}$ below the right costal margin. Ophthalmological examination revealed bilateral Kayser Fleisher (KF) rings. A 24-hour urinary copper was >x60 the Upper Limit of Normal (ULN).

Peripheral blood film had spherocytes with polychromasia. His S. Copper, ceruploplasmin were normal and liver function tests (LFTs) had slight hyperbilirubinemia with direct fraction $>50 \%$ of the total. Magnetic Resonance Imaging (MRI) had T2/FLAIR hyperintensities over the putamen and caudate nuclei which were highly suggestive of WD. Thus with a Leipzig score of 6 the patient was diagnosed of neuro-wilson and tab pencillamine $(20 \mathrm{mg} / \mathrm{kg} /$ day) was started along with vit A, D, E, K and B6 and zinc. He was advised copper free diet, regular follow-up and family screening.

\section{Case 2}

This was the 8 year old brother of the index patient who was asymptomatic with normal abdominal, neurological and ophthalmological examination initially. LFTs, S. Cu and ceruloplasmin levels were also within limits. 24-hour urinary Copper was x1-2 ULN. However on follow-up 6 months later, still asymptomatic, the child's S.Copper increased, ceruloplasmin decreased (though still within limits) and $>50 \%$ of fractionated bilirubin was direct. 24-hour urinary copper 
was now $>x 5$ ULN. This brought his current Leipzig score to 3 with genetic analysis being unavailable and liver biopsy refused by parents. Due to strong family history and clinical suspicion he was also advised the same treatment as his brother. Regular follow-up revealed markedly increased copper excretion after starting chelation therapy that gradually declined over the next 2 years. Chelation was then stopped for a week before measuring $24 \mathrm{hr}$ urinary copper which was now normal. It was then decided to stop chelation and continue with maintenance therapy only consisting of oral Zinc 20mg thrice a day and monitor his copper excretion three monthly.

\section{Case 3}

This was the 48 year old father of these children who was asymptomatic but had hepatomegaly on examination. He had normal LFTs, S.Copper and S.ceruloplasmin but $24 \mathrm{hr}$ urinary copper was >x12 ULN. KF rings were absent and liver biopsy revealed grade 2 steatosis, but rhodamine stain was negative. Leipzig score (excluding genetic analysis) was 2 and it was decided to closely follow the patient for any signs of the disease while keeping him on maintenance therapy with per-oral Zinc three times a day. Further course of action regarding chelation was to be decided after MRI Brain.

\section{Case 4}

He was the 29-year-old paternal uncle of the index case and was brought to notice due to concerns raised by the family. He had previous history of intermittent jaundice once or twice a year since 16 years of age. At the time of examination, the patient was anicteric but liver was palpable $7 \mathrm{~cm}$ below the right costal margin with a total span of $18 \mathrm{~cm}$. There were no peripheral stigmata of chronic liver disease or signs of neurological illness. Hepatic viral serology was negative. Ceruloplasmin was low and $24 \mathrm{hr}$ urinary copper was $>$ x5 ULN. LFTs were deranged with direct hyperbilirubinemia and ALP: total Bilirubin of $<1$. KF rings were absent and liver biopsy could not be done due to affordability issues. Leipzig score (excluding genetic analysis and liver biopsy) was ${ }^{3}$. The strong family history and presence of symptoms prompted us to start chelation along with vitamins $\mathrm{A}, \mathrm{D}, \mathrm{E}, \mathrm{K}$ and $\mathrm{B} 6$ and zinc.

Coombs was negative and reticulocytosis was absent in all four patients. However, both the index case and his paternal uncle had spherocytes. Their complete blood picture revealed decreased mean corpuscular volume (MCV) and increased mean corpuscular hemoglobin $(\mathrm{MCH})$ and mean corpuscular hemog- lobin concentration (MCHC). Other family members including the mother and 5-year-old sister of index case were also screened and found to have normal labs. Screening was also suggested for extended family members such as the father's other siblings. Water analysis for copper content was unremarkable for the drinking water consumed by this family.

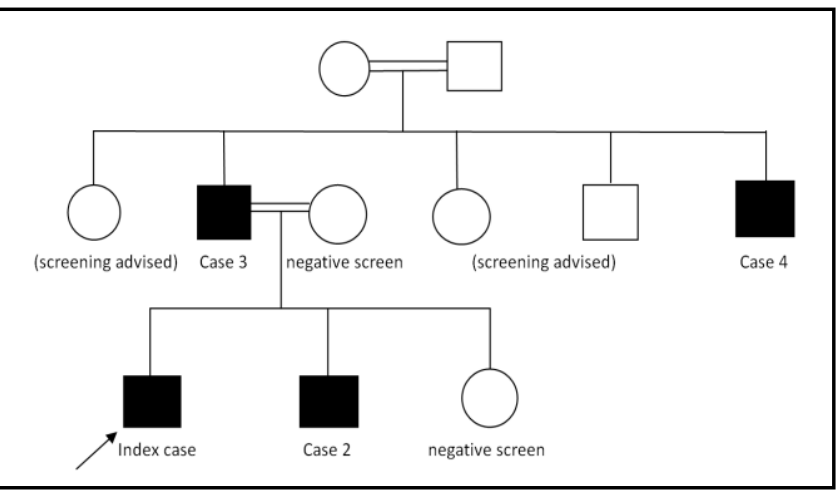

Figure: Pictorial depiction of the pedigree. 2 sisters and 1 brother of case 3 were advised screening (simplified for understanding).

\section{DISCUSSION}

WD is a type of inborn error of metabolism with AR inheritance. A defect in the copper transporting P-type ATPase due to an ATPase 7B mutation leads to impaired excretion of copper in bile ${ }^{3}$. Hepatic wilsonian disease has a varied presentation ranging from acute liver failure, decompensated cirrhosis, acute hepatitis, fatty liver and hepatomegaly and even incidental finding of increased serum transaminases ${ }^{4}$.

Similarly, neurological Wilson may present as worsening school performance, dysarythmia, dysphagia, tremors, dystonia or mood changes ${ }^{5}$. It is often classified into 3 movement disorder syndromes: dystonia, with dystonic postures and choreoathetosis; ataxia, that may be postural with intentional tremors; and parkinsonian, with symptoms of hypokinesia, rigidity and resting tremors ${ }^{6}$.

KF rings, the most characteristic of the ophthalmological presentations, are mostly associated with neuro-wilson, being present in $95 \%$ of such patients. Hematological manifestations include coomb's negative hemolytic anemia and spherocytes on peripheral film. Diagnostic modalities likewise consist of a combination of serum and urine analysis, mutation detection, imaging studies and invasive procedures such as liver biopsy. Typical findings are that of increased free S.Copper and urinary Copper excretion. MRI may reveal the typical double panda sign but can also have 
hyper-intensities over putamen, caudate and thalami nuclei ${ }^{7}$. While S.ceruloplasmin levels maybe falsely elevated or low in a variety of conditions, it is of note to consider the method of measurement. Falsely elevated levels are seen with the immunological-nephelometric assay because it also measures the biologically inactive apoform. Enzymatic assay measuring oxidase activity is a more reliable method ${ }^{8}$.

It is therefore necessary to use scoring systems like the Ferenci score which is similar to the Leipzig score that may increase the sensitivity and specificity of diagnosis to $98.14 \%$ and $96.59 \%$ respectively 9 . Population wide screening is not prudent as criteria set by the European Commission on Public Health and Institute of Human Genetics qualifies WD as a rare disease $^{10}$. With such a wide spectrum of presentation, it is pertinent to keep a high index of suspicion and look for subtle signs in the patients' clinical vignettes as well as the laboratory findings especially amongst family members of previously diagnosed cases.

The only curative option for those with extensive liver involvement is liver transplant (LT) and such patients should be monitored with Kings Wilson Index for timely decision regarding LT $^{11}$. Neurological disease, however, has no cure and treatment options consist of chelators to increase copper excretion and drug agents to reduce gastrointestinal absorption. This in all patients must be complemented with a strict copper free diet, fat soluble vitamins, rehabilitation for speech and movement disorders and regular monitoring to avoid complications of treatment.

\section{Patient's Consent}

Informed consent was obtained from the family to publish this case.

\section{CONFLICT OF INTEREST}

This study has no conflict of interest to be declared by any author.

\section{REFERENCES}

1. Kinnier Wilson SA. Progressive lenticular degeneration: a familial nervous disease associated with cirrhosis of the liver. Brain 1912; 34(1): 295-507.

2. Gitlin JD. Wilson disease. Gastroenterol 2003; 125(6): 1868-77.

3. Bandmann O, Weiss KH, Kaler SG. Wilson's disease and other neurological copper disorders. Lancet Neurol 2015; 14(1): 103-13.

4. Merle U, Schaefer M, Ferenci P, Stremmel W. Clinical presentation, diagnosis and long-term outcome of Wilson's disease: a cohort study. Gut 2007; 56(1): 115-20.

5. Abdel Ghaffar TY, Elsayed SM, Elnaghy S, Shadeed A, Elsobky ES, Schmidt H. Phenotypic and genetic characterization of a cohort of pediatric Wilson disease patients. BMC Pediatr 2011; 11(1): 56-60.

6. Porlas RV Jr, de Castillo LLC, Dioquino CPC. Neurologic Wilson disease: case series on a diagnostic and therapeutic emergency. Dialogues Clin Neurosci 2018; 20(4): 341-45.

7. Czlonkowska A, Tarnacka B, Möller JC, Leinweber B, Bandmann $\mathrm{O}$, Woimant $\mathrm{F}$, et al. Unified wilson's disease rating scale-a proposal for the neurological scoring of Wilson's disease patients. Neurol Neurochir Pol 2007; 41(1): 1-12.

8. Merle U, Eisenbach C, Weiss KH, Tuma S, Stremmel W. Serum ceruloplasmin oxidase activity is a sensitive and highly specific diagnostic marker for Wilson's disease. J Hepatol 2009; 51(5): 925-30.

9. Koppikar S, Dhawan A. Evaluation of the scoring system for the diagnosis of Wilson's disease in children. Liver Int 2005; 25(3): 680-81.

10. Mak CM, Lam CW. Diagnosis of Wilson's disease: a comprehensive review. Crit Rev Clin Lab Sci 2008; 45(3): 263-90.

11. Socha P, Janczyk W, Dhawan A, Baumann U, D'Antiga L, Tanner $S$, et al. Wilson's disease in children: a position paper by the hepatology committee of the european society for paediatric gastro-enterology, hepatology and nutrition. J Pediatr Gastroenterol Nutr 2018; 66(2): 334-44. 